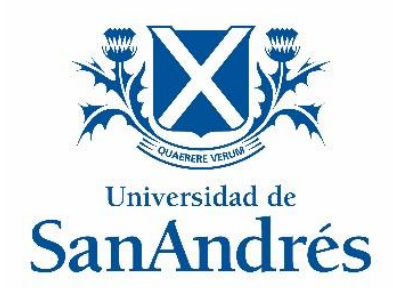

\author{
Universidad de San Andrés \\ Departamento de Economía
}

Maestría en Economía

\title{
Conspicuous Consumption: An Evolutionary Game Theory Explanation
}

Autor: Felipe Montaño Campos

DNI: 94437899

Mentor de Tesis: Federico Weinschelbaum

Victoria, Buenos Aires, Noviembre de 2017 


\title{
Conspicuous Consumption: An Evolutionary Game Theory Explanation
}

\author{
Felipe Montaño Campos*
}

November 2017

\begin{abstract}
The literature has provided a vast amount of empirical evidence consistent with the idea that individuals are affected by their relative position. However, the literature has found it difficult to rationalize this behaviour. In other words, there are few convincing explanations on why individuals are affected by their relative position. My main contribution is to shed light on explaining why individuals have developed relative concerns preferences, inducing them to consume conspicuously. I propose a model of evolutionary game theory where individuals may choose to consume conspicuously, in order to achieve social capital and thus gain a better relative position, or not. I show that conspicuous consumption was constituted and Evolutionary Stable Strategy (ESS) and is asymptotically stable in the Replicator Dynamic, since conspicuous consumption confers individuals an evolutionary advantage through increases in mating opportunities, wealth, power and allies.
\end{abstract}

JEL Classification Codes: C73, D03, I31

Keywords: Relative Concerns, Conspicuous Consumption, Evolutionary Games

\footnotetext{
*Universidad de San Andrés, jmontanocampos@udesa.edu.ar. I would like to express my sincere gratitude to my advisor Prof. Federico Weinschelbaum for the support, guidance and motivation in writing this thesis. The usual disclaimer applies.
} 


\section{Introduction}

There is a consensus in the literature that the welfare of individuals is not solely determined by their material circumstances but also depends heavily on their relative position in society. Gains in utility are higher when individuals get higher levels of income and consumption relative to others than when a generalized increase in income and consumption is carried out among all individuals. Similarly, people perceive dis-utility when they are surrounded by others who have more than they do (Carlsson et al., 2007). The idea that relative concerns are important for people is far from new. A vast number of prominent economists (e.g., Adam Smith, Karl Marx, Jhon Maynard Keynes) in the past have considered seriously that people are concerned with their own income and consumption levels relative to that of others. The literature has provided a vast amount of empirical evidence consistent with the idea that individuals are affected by their relative position. However, the literature has found it difficult to rationalize this behaviour. In other words, there are few convincing explanations on why individuals are affected by their relative position. For instance, the canonical work of Rayo and Becker (2007). They argue that the level of happiness that an individual derives from his economic success is usually affected by the success of his peers (i.e., peer comparisons), and they show that this feature is evolutionary advantageous in the sense of improving the individual's ability to propagate his genes. The main contribution of this paper is to provide an alternative explanation on the mechanism underlying relative concerns.

The impact of inequality among individuals' welfare has gained a great deal of attention considering that relative concerns imply that an increase in the income or consumption level of each individual imposes negative externalities on the others (Perez-Truglia, 2013, 2016). For instance, economic of happiness show that happiness increases with relative income, but is independent of absolute income levels (Easterlin, 1974, 1995). ${ }^{1}$ Several studies have found that one's relative position, as well as one's absolute standard of living, is important in determining mortality (Marmot, 2004; Wilkinson and Pickett, 2006). A wide range of choice experiments have provided support for the idea that people's decisions are influenced by relative income (Fennis, 2008). ${ }^{2}$ And more importantly yet, also individuals' consumption patterns are induced by relative concerns since a large amount of total individuals' expenditures is destined to conspicuous consumption, also known as status-consumption (Rege, 2008)..$^{3}$

\footnotetext{
${ }^{1}$ Just the opposite to conventional economic theory where only absolute income matters.

${ }^{2}$ The literature is too large to do more than note some of the prominent alternatives.

${ }^{3}$ The fact that relative income and consumption concerns exist, is important for our understanding of many economic phenomenons, including aggregate consumption and savings patterns (Duesenberry et al., 1949; Basmann et al., 1988), wage formation (Agell and Lundborg, 1995, 2003), labour supply (Neumark and Postlewaite, 1998) and, the demand for risky activities (Becker and Murphy, 2009), among others.
} 
According to the conspicuous consumption theory, people consume highly observable goods to signal that they are wealthy to others. The fact that the information about the value of their income is private and that the individual's status depends upon perceptions of his income among social contacts, induces individuals to consume conspicuously (Bagwell and Bernheim, 1996; Cole et al., 1992).

Nevertheless, signaling wealth -also understood as signaling economic and social status- in a reference group through conspicuous consumption is costly for people and was considered for many years by economists, anthropologists, ecologists, and biologists, an irrational behavior since they didn't consider the intangible social benefits gained through this behaviour. The accumulation of social capital (symbolic capital) is just as "rational" as the accumulation of economic capital, since social capital gives people advantages in the form of additional wealth, power, allies, and marriage partners (Bourdieu, 1977). The apparent paradox of wasteful spending time and wealth is dissolved since the signal value of conspicuous consumption is maintained by its costs, considering these costs as the price for prestige in a reference group that people pay (BliegeBird et al., 2005).

Conspicuous consumption is considered a way to gain social status, since it is desirable for individuals to consume highly observable expensive goods to show a better relative position. Kuhn et al. (2008) offer related evidence on the link between reference-group income and conspicuous consumption. They study a special lottery in the Netherlands that awards prizes to every ticket holder in a randomly selected postal code each week. They show that when many households win the lottery in one postal code, the non-winning households in that neighborhood change their consumption of items that are highly observable in the context of the neighborhood (e.g., major exterior home renovations). Charles et al. (2009) show that an individuals' expenditure on highly observable goods (clothing and cars) is negatively correlated with the mean income in their state of residence. Bursztyn et al. (2017) shows that demand for the platinum card greatly exceeds demand for a nondescript control product with identical benefits, suggesting demand for the pure status aspect of the card. And, Glazer and Konrad (1996) study the signaling value of donations to US universities. They show that for institutions that report the names of donors in donation categories (e.g., \$1000-1999, $\$ 2000-2999)$, most donations within a given category are very close to the lower bound of that category. This pattern is consistent with the idea that donors care about signaling a better relative position (e.g., income or generosity) than others.

It seems plausible to think that only wealthy people follow these consumption patterns since they have the resources. Nevertheless, evidence shows that even low income households also spend a significant amount of their income in highly observable goods (conspicuous consumption). According to Rao (2001a,b) poor families in India, spend $15 \%$ of households' 
total expenditures in highly observable consumption. He argues that observable consumption has two functions: provides a space for maintaining social reputation and it serves as arenas for status-enhancing competitions. Similarly, Banerjee and Duflo (2007) also show evidence of the large spending by the poor in highly observable festivals, and spending less of $1 \%$ of their income on less observable types of entertainment, such as movies, theater and video shows. Finally, a New York Times article ("Moonshine or the Kids") 4 illustrates how poor people in Africa spend a $\$ 10$ per month on cell phones, and about $\$ 12$ per month on the village bar and, can't afford a $\$ 2,50$ per month tuition for their children. In Trigg (2001) owns words "Conspicuous consumption is considered the most important factor in determining consumer behavior, not just for the rich but for all social classes".

Conspicuous consumption is nearly universal in modern societies and not only in them (Pinker, 1997). Since ancient societies where social interactions became more frequent individuals have incurred in this kind of behaviour. In hunting societies to hunt a large animal prey, it is generally assumed that the main benefit accruing to the hunters is nutritional since the hunters acquire more meat with less cost than with alternative prey. Nevertheless, hunters in many cases behaved just the opposite way. A hunter incurred in the cost of getting and consuming a large prey (highly observable good) not because it offered him more nutritional advantages, but because large preys give hunters social and economic status, increasing his accumulation of social capital (Smith et al., 2003). On the other hand, there is a vast literature among evolutionary biologist considering that higher status, or more precisely the costly-behaviour that generates this status, such as conspicuous consumption, is positively correlated with other desirable genetic characteristics that are associated with greater fitness (Smith and Bliege Bird, 2005). We can, then, state that higher status is desirable for individuals, since it increases mating opportunities and so confers and evolutionary advantage. Biological Evolution evidence tells us that, in the process of evolution, initially there was a correlation between male (female) behaviours preferred by the female (male) and the quality of the male (female). This correlation when appreciated by discriminating females (males) can account for the initial attractiveness of the male (female) (Sexual Selection). These particularly behaviours preferred by females (males) increased the Male's (female's) chance of reproduction (fitness), encouraging males(females) to develop them and, once sexual selection began to have its effect ${ }^{5}$ and social interactions became stronger, it generated a rapid chain of events in which the preference itself created a selective pressure which accounts for the exaggerated development of these attractive behaviours (Conspicuous Consumption) (Fisher, 1930). ${ }^{6}$ At the end of the process, the exaggerated sexually selected behaviour are

\footnotetext{
${ }^{4}$ The New York Times, 22 May 2010, by Nicholas D. Kristof.

${ }^{5}$ Greater Fitness for Males and Females.

${ }^{6}$ Being preferences, via genetic inheritance, correlated to this kind of behaviour.
} 
attractive for males (females) since it increases their relative positions among individuals and, in consequence, their chances of reproduction conferring an evolutionary advantage to them (West-Eberhard, 1983). Since strategizing context-dependent behavior is common in human beings and a learning process mechanism operates among them, through evolution the whole population, regardless of their quality, are induced to strategically adopt these desirable behaviours. Sexual Selection, from the beginning of evolution, induced social competition among individuals. Initiating what we know today as Relative Concerns.

Despite the importance of relative concerns in humans' behaviour the theory is still inconclusive in explaining its underlying mechanism. Mostly, the literature has assumed implicitly that individuals are affected by their relative position in their group of reference and has focused on providing evidence under what circumstances this effect induces individuals to change their behaviour. The closest work related to this paper is De Fraja (2009). The paper develops a model an argues that males undertake conspicuous consumption in order to signal their quality to females, and females are more likely to mate with males whose observed consumption is higher. I argue that among evolution the effects of sexual selection became stronger with the increase in social interactions over time, inducing individuals to change the distribution of ideas and strategies adopted. Initially conspicuous consumption behavior was induced by a small population of high quality individuals increasing the probability of mating. Nevertheless, since strategizing context-dependent behavior is common in individuals and a learning process mechanism operates on the potential benefits of conspicuous consumption, the whole population, regardless their gender and quality, were induced to strategically adopt this behavior developing relative concerns preferences.

To the best of my knowledge this is the first paper that provides a possible explanation on why individuals have developed relative concerns preferences, inducing them to consume conspicuously. I propose a model of evolutionary game theory where individuals may choose to consume conspicuously, in order to achieve social capital, or not to consume conspicuously. The individuals' payoff depends on the amount of social capital achieved, the dis-utility of relative concerns and the cost to consume conspicuously. Under a set of reasonable assumptions I show that conspicuous consumption was constituted and Evolutionary Stable Strategy (ESS) and is asymptotically stable in the Replicator Dynamic, since conspicuous consumption confers individuals an evolutionary advantage through increases in mating opportunities, wealth, power and allies.

The rest of the paper is structured as follows. I introduce the concept of evolutionary game theory under social interactions in Section II. I explain the role of status in shaping individuals' preferences in Section III. I present the model in Section IV. Finally, section V concludes. 


\section{An Evolutionary Game Theory Approach}

The evolutionary approach to game theory has attracted increasing attention not only in the biological sense but, also in the cultural one. Even though both concepts are related, since cultural evolution determines biological evolution (Ehrlich, 2000; Gifford, 2008), when the word "evolution" is used in a biological sense, the approach is concerned with environments in which behavior is genetically determined, and strategy selection obtains because carriers of different genes differ in reproductive fitness. ${ }^{7}$ However, as in my case, often evolution is not intended to be understood biologically. Our framework intends to explain a "cultural evolution" behavior, where in a population of interacting players, learning process is meant. Individuals observe, learn and imitate each other specific behavior whenever they think it increases her fitness.

These learning processes may imply that the distribution of ideas and strategies in a population of agents changes over time in a way that is analogous to biological evolution. This means, that individuals are usually not completely committed to just one set of ideas, or to just one way of behaving -consuming behaviour in our case-. Rather, several systems of ideas, or several possible ways of behaving are present in their minds simultaneously. Which of these predominate, and which are given less attention, depends on the experiences, learning processes of the individual and, in the benefits gained through a particular behaviour. In this sense, the change which the "population of ideas" in the decision maker's mind undergoes may be analogous to biological evolution (Börgers and Sarin, 1997).

The way of behaving that predominates is merely determined by its possible benefits gained from it, specially mating opportunities benefits (Sexual Selection). According to a vast and prominent literature in evolutionary theory, individual behaviour is merely determined by its chances of mating (Andersson, 1994). Over the last three decades, the application of sexual selection theory to human behavior has been the greatest success story in evolutionary psychology, and one of the most fruitful and fascinating developments in the human sciences (Miller et al., 1998). Almost any perceivable behavior can function as an indicator, revealing social status, economic status, strength, intelligence, etc. Such indicators are meant to increase mating opportunities, since those characteristics are desirable in any individual and, as confirmed by Iwasa et al. (1991), these indicators can evolve in order to better fit the desirable characteristics that increase individual fitness (Sexual Selection).

Among human history there is plenty evidence on how sexual selection induced and shaped human behaviour. Mate choice patterns could have favored several classes of courtship behavior that function as indicators that demonstrate physical health, energy, social status,

\footnotetext{
${ }^{7}$ The fitness of an individual describes its ability to survive at a high level of capacity over time and/or describes its ability to reproduce completely and evolve over successive generations (Gill and Hevner, 2013).
} 
intelligence, economic status, etc. Robert (1972) provides evidence that parental investment is induced by sexual selection since those are desirable characteristics. Manning and Taylor (2001) provide evidence that the ability in sports, and particularly ability in football, as a proxy for male physical competitiveness, increases fitness inducing males to developed these kind of abilities. Fox (1972) argued that sexual selection would have favored male hunting prowess, leadership, and tool making. Caspari (1972) considered oratory as an arena of male competition and suggested a role for sexual selection in the evolution of language. This hypothesis can explain the mysterious "cultural" capacities that are universally and uniquely developed in humans, such as mentioned before: language, intellectual creativity, intelligence, art, among others behavioral patterns that are induced by mating opportunities (Miller et al., 1998).

Economic behaviour patterns (Individual Preferences) also are going to work as indicators of, mainly, economic status and social status of individuals and, also are going to be influenced by sexual selection. Since social interactions became more frequent, when the establishment of settled agricultural life set the stage for the emergence of more complex societies by creating the larger and denser populations (Drennan and Peterson, 2008), individuals are affected by their relative position in society and, perceive dis-utility when they are surrounded by others who have more than they do (Carlsson et al., 2007). This is a merely result of a decrease in individuals fitness, relative to others, since a worse relative position means lower social and economic status that decreases mating opportunities among individuals. This dis-utility perceived by individuals induces them to change their behaviour and develop certain preferences in order to revert the particular situation. Under this context, conspicuous consumption arises as a merely result of relative concerns among individuals since this change in consumption pattern tries to revert a lower relative position situation. ${ }^{8}$ This kind of consumption is considered a way to gain social status, or to accumulate social capital, in order to give individuals advantages in the form of additional wealth, power, allies, and marriage partners increasing their relative positions among their group of reference and mitigating the negative effect of relative concerns (Bourdieu, 1977). Nevertheless, at the very beginning when this strategy was initially carried out in some individuals, conspicuous consumption initially was considered an irrational behavior since economists, anthropologists and biologist have paid insufficient attention to the intangible social benefits achieved through this behaviour and, have also paid insufficient attention to prestige-related aspects of individual strategizing (BliegeBird et al., 2005). These components of certain economic behaviors may appear to generate benefits to individuals that are un-measurable.

\footnotetext{
${ }^{8}$ It is also possible to understand conspicuous consumption as a way to get a better relative positions among others and, not necessarily counteract the effect of a lower relative position.
} 
In the process of evolution, initially there was a correlation between male (female) behaviours preferred by the female (male) and the quality of the male (female). This correlation when appreciated by discriminating females (males) can account for the initial attractiveness of the male (female). These particularly behaviours preferred by females (males) increased males' (females') fitness, encouraging males (females) to develop them and, once sexual selection began to have its effect and social interactions became stronger, it generated a rapid chain of events in which the preference itself created a selective pressure which accounts for the exaggerated development of these attractive behaviours (Fisher, 1930).

Thus, we can conclude that among evolution the effects of sexual selection became stronger with the increase in social interactions over time, inducing individuals to change the distribution of ideas and strategies adopted. Initially conspicuous consumption behavior was induced by a small population of high quality individuals increasing their mating opportunities. Nevertheless, since strategizing context-dependent behavior is common in individuals and a learning process mechanism operates on the potential benefits of conspicuous consumption, the whole population -regardless of their quality- are induced to strategically adopt this behaviour given that the social intangible benefits outweigh monetary and non monetary costs. This is how conspicuous consumption went from being a mutant strategy to be constituted an Evolutionary Stable Strategy, and how individuals developed preferences for conspicuous consumption.

\section{The Role of Status in Shaping Individuals' Prefer- ences}

Darwin himself noted that a "a proud man with high status exhibits his sense of superiority over others by holding his head and body erect". A century later, studies would show that posture becomes straighter immediately after social triumph. Also, anthropologist George Peter Murdock published an essay called "The Common Denominator of Cultures", in which he ventured that "status differentiation" was a human universal (Wright, 2010). This provides straight evidence that social distinction or status is crucial in the dynamic of culture and social life since it is recognized as a powerful motivation for human conduct and is universal in societies (Bourdieu, 1979; Veblen, 1899).

Darwin (1883) was one of the first prominent articles that provides an explanation on individuals status concerns. He introduced sexual selection as a selection mechanism that induces individuals to be or appear a more attractive mating partner than their same sex competitors. This causes individuals to be concerned about their status, since it makes them 
more or less competitive among mating competitors, and therefore encourage individuals to develop certain characteristics or behaviors that provides them a higher relative position (e.g., status) among peers.

Sexual selection from the beginning of evolution induce social competition among individuals, initiating what we know today as relative concerns, and induced individuals to develop preferences for status. There is much empirical and theoretical evidence of the importance of social status and how these preferences induced a change in individuals behavior under several contexts and how it influences individuals' well-being. Among them we can mention Happiness Studies, Stated Preferences, Revealed Preferences and, Medical and Biological Evidence.

Among Happiness Studies we can mention the pioneered research by Easterlin (1974, 1995) who studies how status concerns affect the happiness of individuals. Evidence comes from national and international happiness comparison, for which respondents are asked to state their level of happiness or subjective well-being on an index scale. Results are surprising, one finds no time trend in average happiness in spite of an (constant dollar) increase of median national family income of $40 \%$ in USA. ${ }^{9}$ Nevertheless, there is a strong correlation between relative income and stated happiness within country comparisons. For USA, European Union and Switzerland higher income-status families report on average higher happiness that poorer families. The correlation between stated happiness and relative income is both relatively strong and very significant(Easterlin, 1974, 1995; O'cass and McEwen, 2004; Frey and Stutzer, 1999).

The Stated Preference literature asks individuals to state their preferences before a hypothetical choice problem. Individuals have to choose the option that they believe would maximize their utility. Solnick and Hemenway (1998) ask individuals to choose between two states: a "relative case " $\mathrm{A}$, in which respondent is better off in relative terms (compared to case B) but worse off in absolute terms, and "absolute case" B, in which one is better off in absolute terms, but worse off in relative terms than the others. For Example:

- A: Your current yearly income is $\$ 10,000$; others earn $\$ 2,000$

- B: Your current yearly income is $\$ 20,000$; others earn $\$ 50,000$

Results are consistent with status concerns. Up to $80 \%$ of the respondents prefer the relative case. Individuals care about their relative position among peers.

Among Revealed Preferences papers we can mention the most important ones. Glazer and Konrad (1996) argues that charitable giving is motivated by status concerns. They found

\footnotetext{
${ }^{9}$ One finds similar results for nine European countries between 1973-1989. In Japan, among 1958-1987, the real per capita income increased five times. However, the average level of happiness remained unchanged (Truyts, 2010)
} 
that status concerns is shown by donation records of universities that report the names of donors in categories (e.g. \$100-\$200,\$201-\$300). Glazer and Konrad observe that mean donations within each category are very close to the category lower bound. This pattern is consistent with the idea that donors do not only care about helping universities, but being perceived richer and with a higher relative position. Kosicki (1987) explains how the fact that saving rates increase with long-run income can easily be rationalized and well predicted by a simple model with relative concerns -contrary to permanent income and life cycle models-. Also, Stark and Taylor (1991) provides evidence that, if absolute income is controlled for, relative income is important in explaining migration decisions.

Finally, there is Medical and Biological evidence that testifies how social status induces something real to happen in both the human and primate brain and body. For instance, Long et al. (1982) finds evidence that people show a higher heart rate and blood pressure when confronted with an experimenter who bear signs of high status (suit, name tag, formal language).

The above literature provides direct evidence that individuals are concerned about their status (e.g. relative position) and, therefore, are induced in behaviors that provide them a higher status among peers. My contribution is to propose a model that explains why individuals have developed relative concerns preferences, that induced them in conspicuous consumption.

\section{The Model}

Two concepts that have been central to the study of evolutionary models are the Evolutionary Stable Strategy (ESS) and the Replicator Dynamic (RD). While the ESS refers to a static concept of equilibrium, the replicator dynamic refers to a dynamic concept of equilibrium. On one hand, Evolutionary Stable Strategy is robust to evolutionary selection pressures in an exact sense (Smith and Price, 1973; Smith, 1974, 1982). It essentially requires that the strategy be robust when it is invaded by a small population playing a different strategy. Suppose that individuals are repeatedly drawn at random from a large population to play a symmetric two-person game, and suppose that initially all individuals are "programmed" to play a certain pure or mixed strategy. The incumbent strategy is said to be evolutionary stable if, for each mutant strategy, there exists a positive invasion barrier such that if the population share of individuals playing the mutant strategy falls below this barrier, then the incumbent strategy earns a higher payoff than the mutant strategy (Weibull, 1997). The ESS concept is a refinement of Nash equilibrium since every ESS is Nash but, not every Nash is a ESS (Fudenberg and Levine, 1998). However, as with Nash Equilibrium, the concept of 
ESS is silent about the underlying dynamics that give rise to the equilibrium. Instead, asks whether, once reached, a strategy is robust to mutant strategies (evolutionary pressures). An important advance, the Replicator Dynamic, clarifies the dynamic underlying an ESS. The Replicator Dynamic captures, in a simple model, the idea that fitter strategies will have a higher growth rate in the population (Dhami, 2016). Essentially, it assumes that the population playing a particular strategy grows in proportion to how well that strategy is doing relative to the mean population payoff. This means that population playing a strategy $S$ will exponentially grow if the expected payoff of using $S$ is higher than the average expected payoff in the population. The asymptotically stable steady state in the replicator dynamic, as ESS, is a refinement of Nash equilibrium since every asymptotically stable steady state is Nash, but not vice versa (Bomze, 1986). The criterion of evolutionary stability refers implicitly to a close connection between the payoff in the game and the spreading of a strategy in a population. As mentioned before, the higher the payoff of a particular strategy the faster the learning processes mechanism operates, spreading this particular strategy among players. ${ }^{10}$

One goal of the literature on evolution is to establish more closely the connection between replicator dynamics and the ESS concept. In general, an evolutionary process combines two basic elements: a mutation mechanism that provides variety and a selection mechanism that favors some varieties over others. While the criterion of evolutionary stability highlights the role of mutations, the replicator dynamics highlights the role of selection. There is a key link between static and dynamic evolutionary approaches. In particular, every ESS is an asymptotically stable steady state of the replicator dynamics (Taylor and Jonker, 1978; Hofbauer et al., 1979; Zeeman, 1980). Thus, we can state that,

$$
E S S \Rightarrow \text { Asymptotically Stable Steady State in The RP } \Rightarrow N E
$$

My main result from the model implies that conspicuous consumption, driven by relative concerns and sexual selection, constitutes an Evolutionary Stable Strategy among Evolution. Since the dynamic underlying the ESS is the Replicator Dynamic, I can conclude that conspicuous consumption confers individuals an evolutionary advantage (i.e. individuals get a higher expected payoff than the average) inducing the whole population to adopt this strategy through the learning mechanism.

\section{IV.I A Conspicuous Consumption Model}

I consider a population of individuals whose members are repeatedly randomly matched in pairs. The population is large enough that the probability of an individual being matched

\footnotetext{
${ }^{10}$ The payoffs in the game are supposed to represent the gain in biological fitness.
} 
with the same opponent more than once is negligible. The set of possible models of behavior of every individual is the same, and the consequence of an interaction for an individual depends only on its action and that of its opponent, not on its name. I model the interaction between each pair of individuals as a two-player symmetric strategic game in a single homogeneous population. In this context, such a game consists of a common set $S$ of strategies and a payoff function $U$. An individual may choose to consume conspicuously, denoted by $C$, or not to consume conspicuously, denoted by $\bar{C}$. For any pair of strategies $(C, \bar{C})$, the payoff $U(C, \bar{C})$ is interpreted as the fitness of an individual that takes the action $C$ when its opponent takes the action $\bar{C}$. The fitness (or expected payoff) of each individual in all the possible situations are as follows:

$$
\begin{aligned}
& U_{i}(C, C)=\frac{1}{2} k-c \\
& U_{i}(C, \bar{C})=k-c \\
& U_{i}(\bar{C}, C)=-\gamma \\
& U_{i}(\bar{C}, \bar{C})=\bar{k}
\end{aligned}
$$

Variable $k$ refers to the total endowment of social capital distributed among the twoplayers each round of the game. Each individual, of course, intends to appropriates as much $k$ as possible each game since social capital gives people advantages in the form of additional wealth, power, allies, and economic status. Increasing individuals' relative position and therefore their fitness. The variable $c$ is the cost of consume conspicuously. The parameter $\gamma$ captures the absolute relative concerns dis-utility. The higher the dis-utility to be surrounded by others who have more than you do, the higher the value of the parameter $\gamma$. Finally, $\bar{k}$ captures the social capital of reserve. It could be interpreted as the standard gaining in social capital by each player when there is no changes in consumption patterns among players so, no social interaction effect takes place. I assume that the total possible gainings in social capital are big enough that $k>2 c$ and, whenever an opponent is playing $\bar{C}$, the individual also plays $\bar{C}$ in order to avoid potential psychological and monetary costs $(\bar{k}>k-c)$. In other words, whenever player $i$ plays $C(\bar{C})$, player $j$ plays also $C(\bar{C})$. The game between each pair of individuals in its normal form is as in table 1 ,

Conspicuous consumption is considered a way to gain economic and social status, since is desirable for individuals to consume highly observable expensive goods to show a better relative position among their group of reference. For instance when player 1 consumes conspicuously and player 2 does not. Player 1 gets a positive fitness equal to the total amount of social capital disputed in the game minus its respective cost. This positive fitness corresponds to a higher relative position for player 1 compared to player 2. On the other hand 
Table 1: Conspicuous Consumption Game

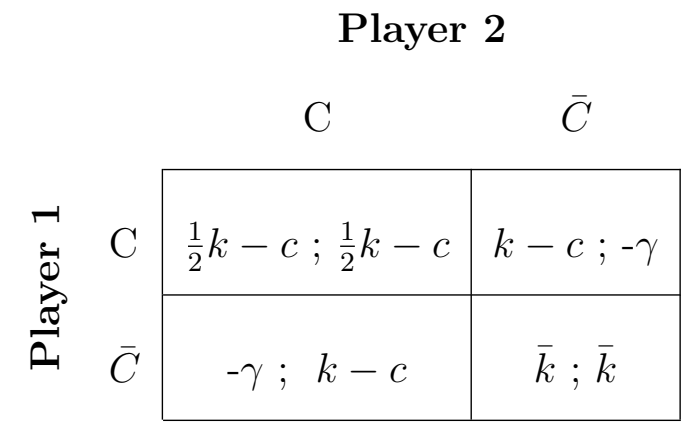

player 2, obviously, gets a worse relative position compared to player 1, which means lower economic and social status and, lower fitness captured by his expected payoff $-\gamma$ (relative concerns dis-utility parameter). Whenever the two players consume conspicuously, the total endowment of social capital is distributed equally to both players. Of course, each player pays the respective cost $c$. Finally, both players get a fitness equal to the social capital of reserve when they consume not conspicuously.

Two pure strategy Nash equilibriums arise in our particular framework: $(C, C)$ and $(\bar{C}, \bar{C}) .{ }^{11}$ It is plausible to think that in the initial conditions individuals were "programmed" to play $(\bar{C}, \bar{C})$ since in early societies individuals used to live in very small nomad societies, where individuals used to move every short period of time, not settling themselves in one fixed place. Thus, the probability of interacting with big amounts of individuals and establish social relationships was very low, arising no social effects on them (Asad, 1978). In other words, there was no need in changing their consumption patterns in order to revert any potential relative concern. Nevertheless as time passed by, social and environmental conditions changed. The establishment of settled agricultural life set the stage for the emergence of more complex, centralized and, hierarchical societies where social interactions became a thing of every day (Drennan and Peterson, 2008). Social interactions induced individuals to change their patterns of consumption in order to compete for a status that provides a better relative position among individuals. Our main objective is to determine under what conditions $C$ is constituted an Evolutionary Stable Strategy among evolution and to determine whether or not is asymptotically stable.

Let's assume, for all the reasons provided above, that initially individuals were "programmed" to play $\bar{C}$. Thus, I first aim to find under what conditions strategy $\bar{C}$ is successfully invaded by mutant strategy $C$. Suppose the population contains the small fraction $\varepsilon_{c}$ of mutants who play the strategy $C$. The population is large, so the probability of any

11 There is one Nash Equilibrium in mixed strategies: $p=\frac{\bar{k}-(k-c)}{\gamma+\bar{k}-\frac{1}{2} k}$. 
individual being matched with a mutant is $\varepsilon_{c}$ and, the probability of being match with a non-mutant (players that play strategy $\bar{C}$ ) is $1-\varepsilon_{c}$. The fitness of a normal individual is

$$
\begin{aligned}
U(\bar{C}) & =\varepsilon_{c}(-\gamma)+\left(1-\varepsilon_{c}\right)(\bar{k}) \\
U(\bar{C}) & =\bar{k}-\varepsilon_{c} \gamma-\varepsilon_{c} \bar{k}
\end{aligned}
$$

The fitness of a mutant individual that plays $C$ is

$$
\begin{aligned}
U(C) & =\varepsilon_{c}\left(\frac{1}{2} k-c\right)+\left(1-\varepsilon_{c}\right)(k-c) \\
U(C) & =k-c-\frac{1}{2} \varepsilon_{c} k
\end{aligned}
$$

If we compare fitness of strategy $C$ versus fitness of strategy $\bar{C}$ we get the following inequality

$$
\begin{aligned}
U(C) & >U(\bar{C}) \\
\varepsilon_{c} & >\frac{\bar{k}-(k-c)}{\gamma+\bar{k}-\frac{1}{2} k}=\varepsilon_{1}^{T}
\end{aligned}
$$

My parameter of interest is $\varepsilon_{1}^{T} .{ }^{12}$ This is the threshold that determines whether strategy $C$ successfully invades $\bar{C}$ or not. Intuitively, inequality (1) tells us that if the fraction of mutants $\varepsilon_{c}$ is greater than $\varepsilon_{1}^{T}$, strategy $\bar{C}$ is not Evolutionary Stable Strategy since it is successfully invaded by strategy $C$. Under this circumstance strategy $C$ provides individuals a better fitness inducing all of them to adopt it (Replicator Dynamic).

It is interesting to analyze how $\varepsilon_{1}^{T}$ is determined given the exogenously parameters considered in the game (Table 2). As expected, the higher the social capital disputed each game (k) the lower the threshold of mutants required to successfully invade strategy $\bar{C}$. In other words, few individuals playing strategy $C$ are required to induce the whole population to play that strategy when $k$ increases $\left(\frac{\partial \varepsilon_{1}^{T}}{k}<0\right)$. This result is very intuitive, individuals are encouraged to play strategy $C$, ceteris paribus, since the potential gainings in relative position among individuals are higher than when playing $\bar{C}$. Similarly, a smaller fraction of mutants $\varepsilon_{c}$ are required to successfully invade the incumbent strategy when the absolute value of relative concerns parameter increases $\left(\frac{\partial \varepsilon_{1}^{T}}{\gamma}<0\right)$. The dis-utility driven by relative concerns gets higher in absolute value whenever individuals get a worse relative position. Thus, in order to revert this situation individuals are encouraged to change their consumption patterns and play $C$ to improve their relative position. This implies that individuals are induced to adopt strategy $C$ since this strategy increases their fitness. On the other hand, when the cost of

\footnotetext{
${ }^{12}$ A positive number $\in(0,1)$ (See Appendix A.I).
} 
consume conspicuously gets higher, is less likely to invade successfully strategy $\bar{C}$ since it is required a higher fraction of mutants $\left(\frac{\partial \varepsilon_{1}^{T}}{c}>0\right)$. It is obvious that individuals will feel less encouraged to follow strategy $C$ if its cost is really high. Finally, the higher $\bar{k}$ the higher fraction of mutants needed to invade the incumbent strategy $\left(\frac{\partial \varepsilon_{1}^{T}}{k}>0\right)$. If the standard gaining in social capital $(\bar{k})$, when no social interactions arise, is increased. Individuals feel less likely to adopt strategy $C$ since they avoid to engage themselves in status competition because of the possible monetary and psychology costs. This is why is more difficult to successfully invade strategy $\bar{C}$ since individuals are fine with Status-quo (See Appendix A.II for the calculations of the derivatives).

Table 2: Net-Effect on the fraction of mutants $\varepsilon_{1}^{T}$

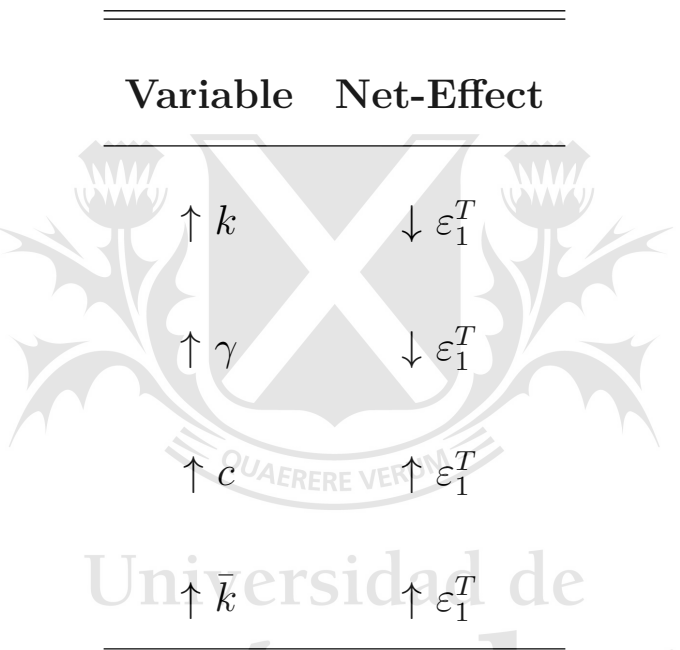

Given the underlying mechanism of the game explained above. We can now analyze how the initial conditions of the game are affected when social and environmental conditions change. The emergence of more complex, centralized and, hierarchical societies increase exponentially social interactions between individuals. This would imply only an increase in $k$ and $\gamma$ in my framework since individuals are competing for status and try to improve their relative position to mitigate the relative concern effect. Under these circumstances strategy $C$ could successfully invades strategy $\bar{C}$. Individuals will feel encouraged to change their consumption patterns in order to improve in fitness and mitigate relative concerns effect.

In other words, the increase in $k$ and $\gamma$ will induce the whole population to follow strategy $C$ since the mutant strategy earns a higher payoff than the incumbent strategy. Up here, It can not be stated that strategy $C$ is an ESS. It was proved that it successfully invades strategy $\bar{C}$. To determine whether this strategy is an ESS it needs to resist mutants. Otherwise the equilibrium would not be stable since players would go from strategy $\bar{C}$ to strategy $C$, in the next period from strategy $C$ to strategy $\bar{C}$ and, so on. 
The new incumbent strategy and mutant strategy is $C$ and $\bar{C}$, respectively. I now aim to prove that to consume conspicuously repeal invaders and so it is constituted as an ESS. Let's assume that the population contains a small fraction $\varepsilon_{\bar{c}}$ of mutants who play the strategy $\bar{C}$. The probability of any individual being matched with a mutant is $\varepsilon_{\bar{c}}$ and the probability of being matched with non-mutant is $1-\varepsilon_{\bar{c}}$. The fitness of a normal individual is

$$
\begin{aligned}
& U(C)=\left(1-\varepsilon_{\bar{c}}\right)\left[\frac{1}{2} k-c\right]+\varepsilon_{\bar{c}}[k-c] \\
& U(C)=\frac{1}{2} k+\frac{1}{2} k \varepsilon_{\bar{c}}-c
\end{aligned}
$$

The fitness of a mutant individual that plays $\bar{C}$ is

$$
\begin{aligned}
U(\bar{C}) & =-\gamma\left(1-\varepsilon_{\bar{c}}\right)+\varepsilon_{\bar{c}} \bar{k} \\
U(\bar{C}) & =-\gamma+\gamma \varepsilon_{\bar{c}}+\varepsilon_{\bar{c}} \bar{k}
\end{aligned}
$$

If we compare fitness of strategy $C$ versus fitness of strategy $\bar{C}$ we get the following inequality

$$
\begin{aligned}
U(C) & >U(\bar{C}) \\
\varepsilon_{2}^{T} & =\frac{\frac{1}{2} k-c+\gamma}{\gamma+\bar{k}-\frac{1}{2} k}>\varepsilon_{\bar{c}}
\end{aligned}
$$

The threshold that determines whether strategy $\bar{C}$ invades $C$ or not is $\varepsilon_{2}^{T} .{ }^{13}$ Intuitively, this parameter establishes the minimum fraction of mutants needed to successfully invade the incumbent strategy. The higher $\varepsilon_{2}^{T}$, the more robust is strategy $C$ since it is required a bigger fraction of mutants. In other words, strategy $C$ resists more to evolutionary pressures.

As in the previous case, it is interesting to analyze how $\varepsilon_{2}^{T}$ is determined given the exogenously parameters considered in the game (Table 3 ). When $k$ increases $\varepsilon_{2}^{T}$ also increases $\left(\frac{\partial \varepsilon_{2}^{T}}{k}>0\right)$. This implies that strategy $C$ is more resistant to evolutionary pressures when the potential gainings in relative positions are high, since a higher fraction of mutants are required to carry out a successful invasion. This result is very intuitive, individuals will feel more strongly convinced consuming conspicuously since its expected payoff increases compared to strategy $\bar{C}$. Analogously, there is a positive relation between $\gamma$ and $\varepsilon_{2}^{T}$ in the game $\left(\frac{\partial \varepsilon_{2}^{T}}{\gamma}>0\right)$. The higher the dis-utility to be surrounded by individuals who have more than you do, the more encouraged you feel to play strategy $C$ in order to revert the situation by gaining some relative position among individuals. Thus, strategy $C$ becomes more resistant to evolutionary pressures. On the contrary, there exists a negative relation

\footnotetext{
${ }^{13}$ A positive number $\in(0,1)$ (See Appendix A.III).
} 
between $c$ and $\bar{k}$ with the minimum fraction of mutants needed to successfully invade the incumbent strategy $\left(\frac{\partial \varepsilon_{2}^{T}}{c}<0 ; \frac{\partial \varepsilon_{2}^{T}}{k}<0\right)$. It is easier to invade the incumbent strategy since less individuals are required playing $\bar{C}$. The expected payoff of $C$ decreases since its cost is higher and the gainings in social capital to perform $\bar{C}$ increase. Thus individuals feel more likely to not consume conspicuously (See Appendix A.IV for the calculations of the derivatives).

Table 3: Net-Effect on the fraction of mutants $\varepsilon_{2}^{T}$

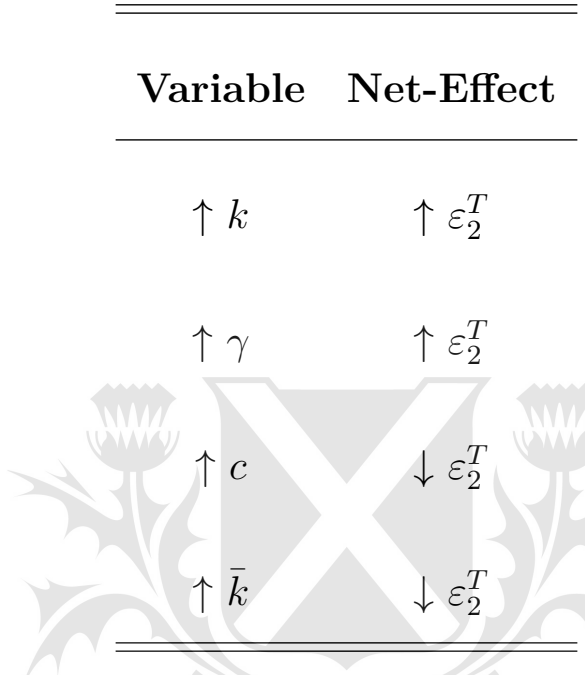

Under my approach I analyze the effect of a discreet increase in social interactions among individuals and, its main implications in their behavior. An increase in $k$ and $\gamma$ implies that strategy $C$ is more resistant to evolutionary pressures since the whole population adopts this strategy and the expected payoff of the mutant strategy gets lower. Under these circumstances strategy $C$ not only successfully invades strategy $\bar{C}$ but, also constitutes and Evolutionary Stable Strategy since it repeals successfully invaders. ${ }^{14}$ As mentioned before the criterion of evolutionary stability highlights the role of mutation (static approach), while the replicator dynamics highlights the underlying mechanism under the strategy that resist evolutionary pressures (dynamic approach). The replicator dynamics is formalized as a system of ordinary differential equations that do not include any mutation mechanism at all. Instead robustness against mutations is indirectly taken care of by the way of dynamic stability criteria. The main objective now is to prove that $C$ is dynamic stable, otherwise there could arise a change in equilibrium by simply changing the payoffs slightly. In other words, it is needed to prove that regardless the initial conditions in the fraction of individuals playing $C$, over time all the population will always end up playing $C$ (See Figure 1 in Appendix

\footnotetext{
${ }^{14}$ Note that my theoretical approach contemplates the special case where strategy $C$ is a strictly dominant strategy. This is the case where $k-c>\bar{k}$ and, thus $\varepsilon_{1}^{T}=0$ and $\varepsilon_{2}^{T}=1$. Implying that strategy $C$ is never invaded by strategy $\bar{C}$ and is always constituted an ESS.
} 
A.VI). ${ }^{15}$

Following Fudenberg and Levine (1998) let's define the dynamic replicator, how it might be interpreted and whether or not a strategy is asymptotically stable or not. Let $\theta_{t}(s), s \in S$, be the fraction of player using pure strategy $s$ at date $t$. Then the expected payoff of using pure strategy $s$ at date $t$ is $u_{t}(s)=\sum_{s^{\prime}} \theta_{t}\left(s^{\prime}\right) u_{t}\left(s, s^{\prime}\right)$, and the average expected payoff in the population is $\bar{u}=\sum_{s} \theta(s) u_{t}(s)$. As before, each individual is genetically programmed to play some pure strategy, and that this programming is inherited. Finally the net reproduction rate of each individual is proportional to its score in the stage game. This leads to the continuous-time dynamic system:

$$
\dot{\theta}_{t}(s)=\theta_{t}(s)\left[u_{t}(s)-\bar{u}_{t}\right]
$$

Equation (3) says that strategies with positive scores have positive net growth rates. Thus, if a strategy is doing better than the rest, the fraction of the population playing this strategy is constantly increasing. Given our framework the resulting replicator dynamics are given by

$$
\begin{aligned}
& \dot{\theta}_{t}(C)=\theta_{t}(C) \theta_{t}(\bar{C})\left[\theta_{t}(C)\left(\frac{1}{2} k-c+\gamma\right)+\theta_{t}(\bar{C})(k-c-\bar{k})\right] \\
& \dot{\theta}_{t}(\bar{C})=\theta_{t}(\bar{C}) \theta_{t}(C)\left[\theta_{t}(C)\left(-\frac{1}{2} k+c-\gamma\right)+\theta_{t}(\bar{C})(-k+c+\bar{k})\right]
\end{aligned}
$$

Linearizing at the equilibrium $(\theta(\bar{C})=1, \theta(\bar{C})=0)$, we find that the Jacobian is

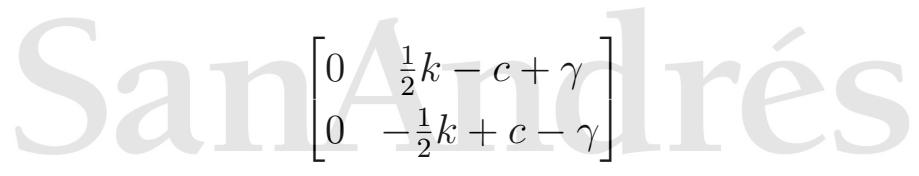

The eigenvalues of this matrix are the solutions of $-\lambda\left(-\frac{1}{2} k+c-\gamma\right)+\lambda^{2}=0$ or, $\lambda\left[\lambda-\left(-\frac{1}{2} k+\right.\right.$ $c-\gamma)]=0$, and hence have strictly negative real part (See Appendix A.V). This means that the continuous-time dynamic system is asymptotically stable steady state when the whole population adopts strategy $C .^{16}$

It can be concluded, then, that among evolution strategy $C$ not only successfully invaded strategy $\bar{C}$ but, also constituted an Evolution Stable Strategy since it repeals successfully invaders. The dynamic underlying the resistance of evolutionary pressures follows as the Replicator Dynamic. Thus, conspicuous consumption, asymptotically stable in the replicator dynamic, confers individuals an evolutionary advantage (i.e. individuals get a higher expected

\footnotetext{
${ }^{15}$ The system of ordinary differential equations will not be degenerated. This implies that any deviation from the equilibrium will eventually return to the stable condition.

${ }^{16}$ The result is consistent with Taylor and Jonker (1978), Hofbauer et al. (1979), and Zeeman (1980).
} 
payoff than the average) inducing the whole population to adopt this strategy over time.

\section{Conclusions}

It is well known that individuals are concerned about their relative position in society since they perceive dis-utility when they are surrounded by others who have more than they do. This induces individuals to care and try to manipulate their status in order to improve their relative position. A vast literature studies several contexts under which individuals are concerned about their relative position and, therefore, how they are induced to change their conduct. However, there is still a lack of explanation on the mechanism underlying relative concerns. The main contribution of this paper is to provide a possible explanation on why individuals have developed relative concerns preferences that induce them in conspicuous consumption.

Among evolution the effects of sexual selection, that induced individuals to compete in fitness, became stronger with the increase in social interactions over time inducing individuals to change the distribution of ideas and strategies adopted. Individuals were induced in certain economic behaviour patterns, as conspicuous consumption, that work as indicators of status.

Conspicuous consumption strategy, initially, was induced by a small mutant population of high quality individuals. However, with the increase in the frequency in social interactions and, since strategizing context-dependent behavior is common in individuals and a learning process mechanism operated on the potential benefits of conspicuous consumption, the whole population, regardless of their quality, was induced to strategically adopt this behavior in order to get a better fitness and so, a better relative position among individuals.

This is how conspicuous consumption became an evolutionary stable strategy (ESS) over time and since the mechanism underlying the ESS is the Replicator Dynamic, it can be concluded that conspicuous consumption confers individuals an evolutionary advantage since mating opportunities, wealth, power, and, allies are increased (i.e. individuals get a higher expected payoff than the average). 


\section{References}

Agell, J. and Lundborg, P.: 1995, Theories of pay and unemployment: survey evidence from swedish manufacturing firms, The Scandinavian Journal of Economics pp. 295-307.

Agell, J. and Lundborg, P.: 2003, Survey evidence on wage rigidity and unemployment: Sweden in the 1990s, The Scandinavian Journal of Economics 105(1), 15-30.

Andersson, M. B.: 1994, Sexual selection, Princeton University Press.

Asad, T.: 1978, Equality in nomadic social systems? (notes towards the dissolution of an anthropological category), Critique of Anthropology 3(11), 57-65.

Bagwell, L. S. and Bernheim, B. D.: 1996, Veblen effects in a theory of conspicuous consumption, The American Economic Review pp. 349-373.

Banerjee, A. V. and Duflo, E.: 2007, The economic lives of the poor, The Journal of Economic Perspectives 21(1), 141-167.

Basmann, R. L., Molina, D. J. and Slottje, D. J.: 1988, A note on measuring veblen's theory of conspicuous consumption, The Review of Economics and Statistics pp. 531-535.

Becker, G. S. and Murphy, K. M.: 2009, Social economics: Market behavior in a social environment, Harvard University Press.

BliegeBird, R., Smith, E., Alvard, M., Chibnik, M., Cronk, L., Giordani, L., Hagen, E., Hammerstein, P., Neiman, F., Bird, R. et al.: 2005, Signaling theory, strategic interaction, and symbolic capital 1, Current anthropology 46(2), 221-248.

Bomze, I. M.: 1986, Non-cooperative two-person games in biology: A classification, International journal of game theory 15(1), 31-57.

Börgers, T. and Sarin, R.: 1997, Learning through reinforcement and replicator dynamics, Journal of Economic Theory 77(1), 1-14.

Bourdieu, P.: 1977, Outline of a Theory of Practice, Vol. 16, Cambridge university press.

Bourdieu, P.: 1979, La distinctíon: Critique sociale du jugement.

Bursztyn, L., Ferman, B., Fiorin, S., Kanz, M. and Rao, G.: 2017, Status goods: experimental evidence from platinum credit cards, Technical report, National Bureau of Economic Research. 
Carlsson, F., Johansson-Stenman, O. and Martinsson, P.: 2007, Do you enjoy having more than others? survey evidence of positional goods, Economica 74(296), 586-598.

Caspari, E.: 1972, Sexual selection in human evolution, Sexual Selection and the Descent of Man pp. 332-56.

Charles, K. K., Hurst, E. and Roussanov, N.: 2009, Conspicuous consumption and race, The Quarterly Journal of Economics 124(2), 425-467.

Cole, H. L., Mailath, G. J. and Postlewaite, A.: 1992, Social norms, savings behavior, and growth, Journal of Political Economy 100(6), 1092-1125.

Darwin, C.: 1883, The Descent of Man and Selection in Relation to Sex, .

De Fraja, G.: 2009, The origin of utility: Sexual selection and conspicuous consumption, Journal of Economic Behavior \& Organization 72(1), 51-69.

Dhami, S.: 2016, The Foundations of Behavioral Economic Analysis, Oxford University Press.

Drennan, R. D. and Peterson, C. E.: 2008, Centralized communities, population, and social complexity after sedentarization, The Neolithic demographic transition and its consequences, Springer, pp. 359-386.

Duesenberry, J. S. et al.: 1949, Income, saving, and the theory of consumer behavior.

Easterlin, R. A.: 1974, Does economic growth improve the human lot? some empirical evidence, Nations and Households in Economic Growth 89, 89-125.

Easterlin, R. A.: 1995, Will raising the incomes of all increase the happiness of all?, Journal of Economic Behavior 83 Organization 27(1), 35-47.

Ehrlich, P. R.: 2000, Human Natures: Genes, Cultures, and the Human Prospect, Island Press.

Fennis, B. M.: 2008, Branded into submission: Brand attributes and hierarchization behavior in same-sex and mixed-sex dyads, Journal of Applied Social Psychology 38(8), 1993-2009.

Fisher, R. A.: 1930, The genetical theory of natural selection: a complete variorum edition, Oxford University Press.

Fox, R.: 1972, Alliance and constraint: Sexual selection in the evolution of human kinship systems, Sexual Selection and the Descent of Man 1971, 282-331. 
Frey, B. S. and Stutzer, A.: 1999, Measuring preferences by subjective well-being, Journal of Institutional and Theoretical Economics (JITE)/Zeitschrift für die gesamte Staatswissenschaft pp. $755-778$.

Fudenberg, D. and Levine, D. K.: 1998, The theory of learning in games, Vol. 2, MIT press.

Gifford, A.: 2008, Peter j. richerson and robert boyd, not by genes alone: How culture transformed human evolution, Journal of Bioeconomics 2(10), 193-198.

Gill, T. G. and Hevner, A. R.: 2013, A fitness-utility model for design science research, ACM Transactions on Management Information Systems (TMIS) 4(2), 5.

Glazer, A. and Konrad, K. A.: 1996, A signaling explanation for charity, The American Economic Review 86(4), 1019-1028.

Hofbauer, J., Schuster, P. and Sigmund, K.: 1979, A note on evolutionary stable strategies and game dynamics, Journal of Theoretical Biology 81(3), 609-612.

Iwasa, Y., Pomiankowski, A. and Nee, S.: 1991, The evolution of costly mate preferences ii. the'handicap'principle, Evolution pp. 1431-1442.

Kosicki, G.: 1987, A test of the relative income hypothesis, Southern Economic Journal pp. $422-434$.

Kuhn, P. J., Kooreman, P., Soetevent, A. R. and Kapteyn, A.: 2008, The own and social effects of an unexpected income shock: evidence from the dutch postcode lottery, Technical report, National Bureau of Economic Research.

Long, J. M., Lynch, J. J., Machiran, N., Thomas, S. A. and Malinow, K. L.: 1982, The effect of status on blood pressure during verbal communication, Journal of Behavioral Medicine $5(2), 165-172$.

Manning, J. T. and Taylor, R. P.: 2001, Second to fourth digit ratio and male ability in sport: implications for sexual selection in humans, Evolution and Human Behavior 22(1), 61-69.

Marmot, M.: 2004, The status syndrome: how your social standing affects your health and life expectancy, Bloomsbury, London .

Miller, G. F. et al.: 1998, A review of sexual selection and human evolution: How mate choice shaped human nature, Handbook of Evolutionary Psychology: ideas, issues, and applications. Lawrence Erlbaum: New Jersey pp. 87-130. 
Neumark, D. and Postlewaite, A.: 1998, Relative income concerns and the rise in married women's employment, Journal of Public Economics 70(1), 157-183.

O'cass, A. and McEwen, H.: 2004, Exploring consumer status and conspicuous consumption, Journal of Consumer Behaviour 4(1), 25-39.

Perez-Truglia, R.: 2013, A test of the conspicuous-consumption model using subjective wellbeing data, The Journal of Socio-Economics 45, 146-154.

Perez-Truglia, R.: 2016, The effects of income transparency on well-being: Evidence from a natural experiment, Available at SSRN: https://ssrn.com/abstract=2657808 .

Pinker, S.: 1997, How the mind works. 1997, NY: Norton .

Rao, V.: 2001a, Celebrations as social investments: Festival expenditures, unit price variation and social status in rural india, Journal of Development Studies 38(1), 71-97.

Rao, V.: 2001b, Poverty and public celebrations in rural india, The Annals of the American Academy of Political and Social Science 573(1), 85-104.

Rayo, L. and Becker, G. S.: 2007, Evolutionary efficiency and happiness, Journal of Political Economy 115(2), 302-337.

Rege, M.: 2008, Why do people care about social status?, Journal of Economic Behavior $\&$ Organization 66(2), 233-242.

Robert, T.: 1972, Parental investment and sexual selection, Sexual Selection \& the Descent of Man, Aldine de Gruyter, New York pp. 136-179.

Smith, E. A., Bird, R. B. and Bird, D. W.: 2003, The benefits of costly signaling: Meriam turtle hunters, Behavioral Ecology 14(1), 116-126.

Smith, E. A. and Bliege Bird, R.: 2005, Costly signaling and cooperative behavior, Moral Sentiments and Material Interests: the foundations of cooperation in economic life pp. 115148.

Smith, J. M.: 1974, The theory of games and the evolution of animal conflicts, Journal of theoretical biology 47(1), 209-221.

Smith, J. M.: 1982, Evolution and the Theory of Games, Cambridge university press.

Smith, J. M. and Price, G.: 1973, Lhe logic of animal conflict, Nature 246, 15. 
Solnick, S. J. and Hemenway, D.: 1998, Is more always better?: A survey on positional concerns, Journal of Economic Behavior $\&$ Organization 37(3), 373-383.

Stark, O. and Taylor, J. E.: 1991, Migration incentives, migration types: The role of relative deprivation, The economic journal 101(408), 1163-1178.

Taylor, P. D. and Jonker, L. B.: 1978, Evolutionary stable strategies and game dynamics, Mathematical Biosciences 40(1-2), 145-156.

Trigg, A. B.: 2001, Veblen, bourdieu, and conspicuous consumption, Journal of Economic Issues 35(1), 99-115.

Truyts, T.: 2010, Social status in economic theory, Journal of Economic Surveys 24(1), 137169.

Veblen, T.: 1899, The theory ofthe leisure class, New York: The New American Library .

Weibull, J. W.: 1997, Evolutionary game theory, MIT press.

West-Eberhard, M. J.: 1983, Sexual selection, social competition, and speciation, Quarterly Review of Biology pp. 155-183.

Wilkinson, R. G. and Pickett, K. E.: 2006, Income inequality and population health: a review and explanation of the evidence, Social Science \& Medicine 62(7), 1768-1784.

Wright, R.: 2010, The moral animal: Why we are, the way we are: The new science of evolutionary psychology, Vintage.

Zeeman, E. C.: 1980, Population dynamics from game theory, Global theory of dynamical systems, Springer, pp. 471-497. 


\section{A Appendix}

\section{A.I Rate of Mutants playing C}

The rate of mutants is a positive $\in(0,1)$ number

$$
\varepsilon_{1}^{T}=\frac{\bar{k}-(k-c)}{\gamma+\bar{k}-\frac{1}{2} k}
$$

Given our assumptions it is known that the numerator and denominator are positive. To analyze whether this fraction is smaller than 1 , it is needed to prove that

$$
\begin{aligned}
\bar{k}-(k-c) & <\gamma+\bar{k}-\frac{1}{2} k \\
c & <\frac{1}{2} k+\gamma
\end{aligned}
$$

That inequality holds given the assumptions in the model.

\section{A.II The Dynamic Underlying the rate of Mutants playing $C$}

$$
\begin{aligned}
& \frac{\partial \varepsilon_{1}^{T}}{\partial k}=\frac{\frac{1}{2}[\bar{k}-(k-c)]-\left(\gamma+\bar{k}-\frac{1}{2} k\right)}{\left[\gamma+\bar{k}-\frac{1}{2} k\right]^{2} \mathrm{C} e}<0 \\
& \frac{\partial \varepsilon_{1}^{T}}{\partial \gamma}=-\frac{\bar{k}-(k-c)}{\left[\gamma+\bar{k}-\frac{1}{2} k\right]^{2}}<0 \\
& \frac{\partial \varepsilon_{1}^{T}}{\partial c}=\frac{1}{\left[\gamma+\bar{k}-\frac{1}{2} k\right]}>0 \\
& \frac{\partial \varepsilon_{1}^{T}}{\partial \bar{k}}=\frac{\left(\gamma+\bar{k}-\frac{1}{2} k\right)-[\bar{k}-(k-c)]}{\left[\gamma+\bar{k}-\frac{1}{2} k\right]^{2}}>0
\end{aligned}
$$




\section{A.III Rate of Mutants playing $\bar{C}$}

The rate of mutants is a positive $\in(0,1)$ number

$$
\varepsilon_{2}^{T}=\frac{\frac{1}{2} k-c+\gamma}{\gamma+\bar{k}-\frac{1}{2} k}
$$

Given our assumptions it is known that the numerator and denominator are positive. To analyze whether this fraction is smaller than 1 , it is needed to prove that

$$
\begin{gathered}
\frac{1}{2} k-c+\gamma<\gamma+\bar{k}-\frac{1}{2} k \\
k-c<\bar{k}
\end{gathered}
$$

That inequality holds given the assumptions in the model.

\section{A.IV The Dynamic Underlying the rate of Mutants playing $\bar{C}$}

$$
\begin{aligned}
& \frac{\partial \varepsilon_{2}^{T}}{\partial k}=\frac{\frac{1}{2}\left(\gamma+\bar{k}-\frac{1}{2} k\right)+\frac{1}{2}\left(\frac{1}{2} k-c+\gamma\right)}{\left[\gamma+\bar{k}-\frac{1}{2} k\right]^{2}}>0 \\
& \frac{\partial \varepsilon_{2}^{T}}{\partial \gamma}=\frac{\left(\gamma+\bar{k}-\frac{1}{2} k\right)-\left(\frac{1}{2} k-c+\gamma\right)}{\left[\gamma+\bar{k}-\frac{1}{2} k\right]^{2}}>0 \\
& \frac{\partial \varepsilon_{2}^{T}}{\partial c}=-\frac{1}{\left[\gamma+\bar{k}-\frac{1}{2} k\right]}<0 \\
& \frac{\partial \varepsilon_{2}^{T}}{\partial \bar{k}}=-\frac{\frac{1}{2} k-c+\gamma}{\left[\gamma+\bar{k}-\frac{1}{2} k\right]^{2}}<0
\end{aligned}
$$




\section{A.V Dynamic Stability: Replicator Dynamic}

Let's define the payoff of each strategy and the average expected payoff in the population

$$
\begin{aligned}
u(C) & =\theta_{t}(C)\left[\frac{1}{2} k-c\right]+\theta_{t}(\bar{C})[k-c] \\
u(\bar{C}) & =\theta_{t}(C)(-\gamma)+\theta_{t}(\bar{C}) \bar{k} \\
\bar{u} & =\theta_{t}(C) u(C)+\theta_{t}(\bar{C}) u(\bar{C})
\end{aligned}
$$

If I replace (4) and (5) into (6) I get the following equation

$$
\bar{u}=\theta_{t}(C)\left\{\theta_{t}(C)\left[\frac{1}{2} k-c\right]+\theta_{t}(\bar{C})[k-c]\right\}+\theta_{t}(\bar{C})\left\{\theta_{t}(C)(-\gamma)+\theta_{t}(\bar{C}) \bar{k}\right\}
$$

Now let's define the replicator dynamic for strategy $C$

$$
\begin{aligned}
& \dot{\theta}_{t}(C)=\theta_{t}(C)\left[U_{t}(C)-\bar{u}\right] \\
& \dot{\theta}_{t}(C)=\theta_{t}(C) \theta_{t}(\bar{C})\left[\theta_{t}(C)\left(\frac{1}{2} k-c+\gamma\right)+\theta_{t}(\bar{C})(k-c-\bar{k})\right]
\end{aligned}
$$

The replicator dynamic for strategy $\bar{C}$ is as follows,

$$
\begin{aligned}
& \dot{\theta}_{t}(\bar{C})=\theta_{t}(\bar{C})\left[U_{t}(\bar{C})-\bar{u}\right] \\
& \dot{\theta}_{t}(\bar{C})=\theta_{t}(\bar{C}) \theta_{t}(C)\left[\theta_{t}(C)\left(-\frac{1}{2} k+c-\gamma\right)+\theta_{t}(\bar{C})(-k+c+\bar{k})\right]
\end{aligned}
$$

Finally I get the following dynamic system,

$$
\begin{aligned}
& \dot{\theta}_{t}(C)=\theta_{t}(C) \theta_{t}(\bar{C})\left[\theta_{t}(C)\left(\frac{1}{2} k-c+\gamma\right)+\theta_{t}(\bar{C})(k-c-\bar{k})\right] \\
& \dot{\theta}_{t}(\bar{C})=\theta_{t}(\bar{C}) \theta_{t}(C)\left[\theta_{t}(C)\left(-\frac{1}{2} k+c-\gamma\right)+\theta_{t}(\bar{C})(-k+c+\bar{k})\right]
\end{aligned}
$$

Linearizing at the equilibrium $(\theta(C)=1, \theta(\bar{C})=0)$, I find that the Jacobian is

$$
\jmath=\left[\begin{array}{cc}
0 & \frac{1}{2} k-c+\gamma \\
0 & -\frac{1}{2} k+c-\gamma
\end{array}\right]
$$


In order to get the characteristic polynomial

$$
\jmath-\lambda I=\left[\begin{array}{cc}
-\lambda & \frac{1}{2} k-c+\gamma \\
0 & \left(-\frac{1}{2} k+c-\gamma\right)-\lambda
\end{array}\right]
$$

It is possible, then, characterize the characteristic polynomial as follows,

$$
\begin{aligned}
-\lambda\left(-\frac{1}{2} k+c-\gamma\right)+\lambda^{2} & =0 \\
\lambda\left[\lambda-\left(-\frac{1}{2} k+c-\gamma\right)\right] & =0
\end{aligned}
$$

The roots of the characteristic polynomial are,

$$
\lambda_{1,2}=-\left(\frac{1}{2} k-c+\gamma\right)
$$

Since the parenthesis is a strictly positive number, given our assumptions, it can be stated that the dynamic system when the whole population adopts strategy $C$ is asymptotically stable steady state since the roots of the characteristic polynomial are negative real numbers. 


\section{A.VI Replicator Dynamic for Strategy $C$}

Under the Replicator Dynamic every Nash equilibrium is a steady state since all the strategies being played have the same average payoff, so the population shares are constant $\left(\dot{\theta}_{t}(C)=0\right)$ (Fudenberg and Levine, 1998). Under our approach we have two Nash equilibriums in pure strategies, $(C, C)$ and $(\bar{C}, \bar{C})$, and one Nash equilibrium in mixed strategies $p=\frac{\bar{k}-(k-c)}{\gamma+\bar{k}-\frac{1}{2} k}$. As we can see in Figure 1 all these three equilibriums are steady state.

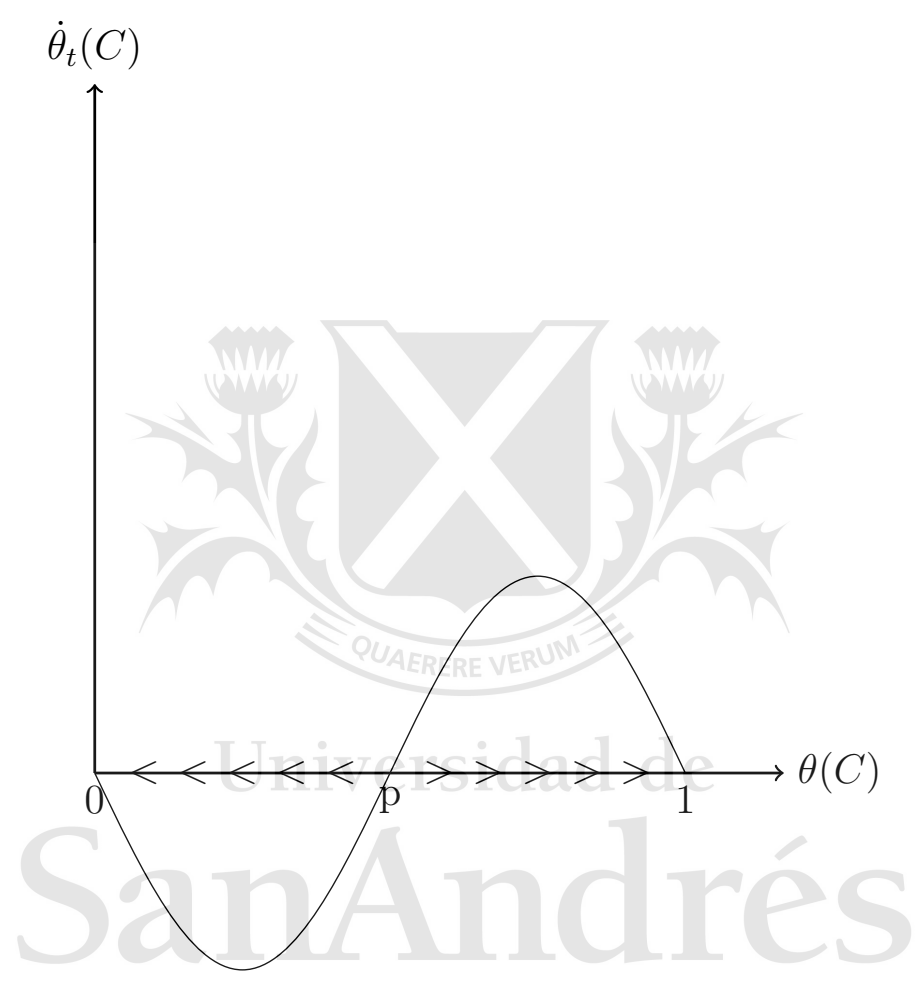

Figure 1: Dynamic Stability

Now let's analyze whether the three steady states (Equilibriums) are asymptotically stable. Let's define the Replicator Dynamic for Strategy C as follows, ${ }^{17}$

$$
\dot{\theta}_{t}(C)=\left[\theta_{t}(C)-\theta_{t}^{2}(C)\right]\left[k-c-\bar{k}+\theta_{t}(C)\left(-\frac{1}{2} k+\gamma+\bar{k}\right)\right]
$$

\footnotetext{
${ }^{17}$ We replaced $\theta_{t}(\bar{C})=1-\theta_{t}(C)$.
} 
Now we calculate $\frac{\partial \dot{\theta}_{t}(C)}{\partial \theta_{t}(C)}$ on the steady states:

$$
\begin{aligned}
& \left.\frac{\partial \dot{\theta}_{t}(C)}{\partial \theta_{t}(C)}\right|_{\theta_{t}(C)=1}=-\left[\frac{1}{2} k+\gamma-c\right]<0 \\
& \left.\frac{\partial \dot{\theta}_{t}(C)}{\partial \theta_{t}(C)}\right|_{\theta_{t}(C)=0}=-[\bar{k}-(k-c)]<0 \\
& \left.\frac{\partial \dot{\theta}_{t}(C)}{\partial \theta_{t}(C)}\right|_{\theta_{t}(C)=p}=\left[\theta_{t}(C)-\theta_{t}^{2}(C)\right]\left(-\frac{1}{2} k+\gamma+\bar{k}\right)>0
\end{aligned}
$$

This implies that steady states $\theta(C)=1$ and $\theta(C)=0$ are asymptotically stable since the derivative is negative. On the other hand the mixed strategy equilibrium is not stable. Again if our parameters of interest $k$ and $\gamma$ increase, $p$ gets lower (In Figure $1 p$ moves to the left) implying that strategy $C$ is more robust. Let's analyze an extreme case, where $k$ and $\gamma$ are high enough that $p$ tends to 0 . This implies that regardless the initial fraction of individuals playing $C$, over time all the population will always end up playing $C$. 